\title{
Microscopic polyarteritis: clinical features and treatment
}

\author{
H. Rodgers, J.A. Guthrie, A.M. Brownjohn and J.H. Turney \\ Regional Renal Unit, The General Infirmary at Leeds, Great George Street, Leeds ¿S1 3EX, UK.
}

\begin{abstract}
Summary: The clinical features, treatment and survival of 36 patients with microscopic polyarteritis diagnosed between 1957-1988 have been reviewed. All had a focal segmental necrotizing glomerulonephritis on renal biopsy with evidence of a small vessel vasculitis. Most had a prodromal illness of less than 1 month. Severity of renal disease varied from microscopic haematuria which has a good prognosis to acute oliguric renal failure which carries a high mortality. The prognosis of microscopic polyarteritis can be improved if the condition is recognized early and treated with steroids and cyclophosphamide.
\end{abstract}

\section{Introduction}

Classical polyarteritis nodosa (PAN) is an inflammatory process involving large and medium sized arteries leading to aneurysm formation. ${ }^{1}$ Microscopic polyarteritis differs from classical PAN clinically, pathologically and prognostically. ${ }^{2,3}$ Microscopic polyarteritis is defined as clinical evidence of a small vessel vasculitis associated with a focal segmental necrotizing glomerulonephritis in the absence of conditions associated with similar renal pathology (Table I). ${ }^{4}$ The classification of renal vasculitis remains unsatisfactory as the clinical and pathological features of these conditions are not distinct and overlap syndromes occur. Microscopic polyarteritis shares several features with Wegener's granulomatosis: both cause a focal segmental necrotizing glomerulonephritis and have positive neutrophil cytoplasmic antibodies (ANCA). ${ }^{5-7}$ Wegener's granulomatosis is characterized by a necrotizing granulomatous vasculitis of the upper and lower respiratory tract with or without renal involvement. The prognosis of both conditions is improved by immunosuppressive therapy. ${ }^{8-20}$

Table I Causes of focal segmental necrotizing glomerulonephritis

Microscopic polyarteritis

Wegener's granulomatosis

Rapidly progressive (crescentic) glomerulonephritis

Systemic lupus erythmatosus

Henoch Schönlein purpura

Churg Strauss syndrome

Nephritis of bacterial endocarditis

Relapsing polychondritis

Correspondence: J.H. Turney, M.A., M.R.C.P.

Accepted: 13 March 1989
Several studies have reviewed the features of polyarteritis. ${ }^{10-21}$ Some include both classical PAN and microscopic polyarteritis, others used a broader definition of microscopic polyarteritis including other arteritides with renal involvement. ${ }^{2,3,10-28} \mathrm{Few}$ have looked specifically at microscopic polyarteritis. ${ }^{4,15}$

\section{Patients and methods}

The clinical records of 36 cases of microscopic polyarteritis diagnosed between 1957 and 1988 were reviewed. All had evidence of small vessel vasculitis and a focal segmental necrotizing glomerulonephritis on renal biopsy. Large vessel disease was not demonstrated by investigation or post-mortem examination. Patients with Wegener's granulomatosis, classical PAN and other vasculitides were excluded. In the study period only two cases of classical PAN were observed. For the purpose of analysis patients were divided into three groups on the basis of presenting renal function: Group A (4 patients) had microscopic haematuria with a stable creatinine of less than $200 \mu \mathrm{mol} / \mathrm{l}$. Group B (8 patients) had deteriorating renal function but did not require dialysis. Group C (24 patients) presented with a rapid decline in renal function requiring dialysis.

The clinical features, treatment and results of laboratory investigations of these patients were examined.

Results

\section{Clinical features}

There were 21 female and 15 male cases of microscopic polyarteritis of age range 27-69 years (median 55

(C) The Fellowship of Postgraduate Medicine, 1989 
years). Most had a non-specific prodromal illness of malaise, myalgia and fever for less than 1 month although in some cases the prodrome was considerably longer (Table II). Renal disease ranged from microscopic haematuria to acute oliguric renal failure with the majority of cases being in the latter group. Myalgia or arthralgia were common but arthritis was not a feature (Table III). Skin manifestations included vasculitic rash, splinter haemorrhages and leg ulceration. Pulmonary haemorrhage occurred in $33 \%$ of cases, usually in association with acute renal failure and had a poor prognosis ( $75 \%$ mortality). Sixty one per cent of patients were hypertensive at presentation; 2 cases were found to have coronary vasculitis at post-mortem examination but clinical cardiac problems were not seen. Peripheral neuropathy was present in 4 cases and mononeuritis multiplex in one. Cerebral vasculitis presented as grand mal fits with deterioration in conscious level and a monoplegia, optic atrophy and sensineural deafness, subarachnoid haemorrhage, and pseudobulbar palsy. Otolaryngological abnormalities were secretory otitis media (1 case) and epistaxis (1 case); both patients had normal nasal biopsies. Conjunctivitis was observed in 2 cases.

Table II Duration of prodrome in 36 patients with microscopic polyarteritis

\begin{tabular}{lc}
\hline Duration & Number \\
\hline$<1$ week & 7 \\
1 week -1 month & 13 \\
$1-3$ months & 7 \\
$3-9$ months & 3 \\
Not known & 6 \\
Total & 36 \\
\hline
\end{tabular}

Table III System involvement at presentation in 36 patients with microscopic polyarteritis

\begin{tabular}{lcc}
\hline & Number & $\%$ \\
\hline Renal & 36 & 100 \\
Hypertension & 22 & 61 \\
(diastolic > 95 mmHg) & & \\
Musculoskeletal & 21 & 58 \\
Skin & 16 & 44 \\
Fever & 14 & 39 \\
Lung (haemorrhage) & 12 & 33 \\
Peripheral nervous system & 5 & 14 \\
Central nervous system & 4 & 11 \\
Otolaryngological & 2 & 5 \\
Ocular & 2 & 5 \\
Cardiac & 2 & 5 \\
\hline
\end{tabular}

\section{Laboratory investigations}

These are summarised in Table IV. Most patients had a normochromic, normocytic anaemia, a neutrophil leucocytosis and the ESR was markedly raised. From 1987 antineutrophil cytoplasmic antibody (ANCA) was measured and was positive in all new cases. Rheumatoid factor was positive in $38 \%$ of cases in whom it was measured. Hepatitis B serology and antinuclear factor were always negative. Serum complement and immunoglobulin levels showed no specific changes.

Table IV Laboratory investigations

\begin{tabular}{lcc}
\hline Haemoglobin & Normal & 9 \\
White cell count & Low & 27 \\
& Normal & 21 \\
& Increased & 15 \\
Eosinophilia & 1 \\
Platelet count & Normal & 19 \\
& Increased & 7 \\
ESR & Decreased & 3 \\
(mm/hour) & $0-20$ & 5 \\
& $21-60$ & 6 \\
Complement C3 & $61-100$ & 5 \\
& $101-120$ & 5 \\
Rheumatoid factor & Normal & 6 \\
& Increased & 13 \\
ANCA & Positive & 8 \\
& Negative & 13 \\
& Positive & 4 \\
& Negative & 0 \\
\hline
\end{tabular}

\section{Cause of death}

There were 21 deaths ( $58 \%)$, the majority (19 deaths) in those patients requiring dialysis. None of the patients with microscopic haematuria and stable renal function died. Figure 1 shows the cause of death against time from presentation to the Renal Unit. The commonest cause of death in the early years of the study ( 9 deaths) was renal failure, but this has not been a cause of death for the last 8 years due to the increased availability of dialysis and the use of immunosuppressive therapy. There were 6 deaths from infection, 4 of which were pneumonia. All infective deaths occurred at least 6 weeks after presentation in immunosuppressed patients.

\section{Treatment}

Fourteen patients received steroids alone; 9 cyclophosphamide and steroids; 2 steroids and azathioprine; and 11 received no specific treatment (Table V).

Group $A$ All 4 patients in this group did well. One patient received no treatment and showed no deterioration in renal function over several months. 

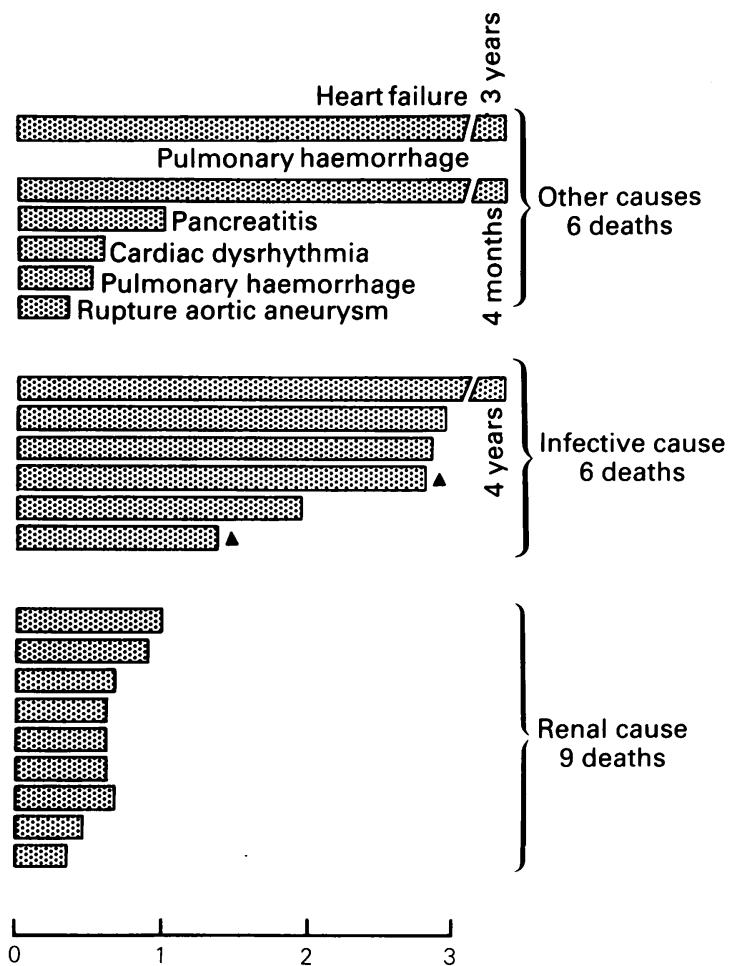

「ime from presentation to death (months)

Figure 1 Microscopic polyarteritis: cause of death. (A Denotes patients in group B. All other deaths were in group C).

Table V Treatment

Steroids +

Supportive Steroids immunosuppression Total

\begin{tabular}{lllll}
\hline $\begin{array}{l}\text { Group A } \\
\text { Number }\end{array}$ & 1 & 2 & 1 & 4 \\
Deaths & 0 & 0 & 0 & 0 \\
& & & & \\
Group B & & & 5 & 8 \\
Number & 0 & 3 & 1 & 2 \\
Deaths & 0 & 1 & & \\
Group C & & & 5 & 24 \\
Number & 10 & 9 & 3 & 19 \\
Deaths & 10 & 6 & & \\
Total & & & 11 & 36 \\
Number & 11 & 14 & 4 & 21 \\
Deaths & 10 & 7 & & \\
\hline
\end{tabular}

Group B All 8 patients received treatment. There were 2 deaths from infection.

Group C Nineteen of the 24 patients died. There were 2 late deaths on this group at 3 and 4 years. All patients who received no immunosuppression died. The mean survival of the untreated group was 15 days (3-35 days). Treatment improved outcome with 5 out of 14 patients surviving.

The patients requiring dialysis had a much higher mortality than those who did not. Five patients in this group survived, 2 required maintenance haemodialysis but the renal function of 2 cases improved such that they did not require further dialysis.

\section{Discussion}

Polyarteritis affecting the kidney is a rare disease but we have seen an increase in referrals in recent years. This may reflect a true increase in incidence as there has not been a comparable increase in other severe nephritides with acute renal failure. When the Renal Unit opened in 1956, patients were referred in established renal failure requiring dialysis. Patients are now seen earlier with a broader spectrum of renal disease ranging from microscopic haematuria with normal renal function to rapidly progressing glomerulonephritis. The variety of coexistent symptoms results in initial presentation to the spectrum of general medical specialities, particularly dermatology, rheumatology, and neurology.

Only cases of biopsy-proven microscopic polyarteritis are included in this study. The sex ratio of 1:1.4 is surprising as other series have reported a preponderance of males., ${ }^{40,13,15,18,24}$ The age distribution is similar to other series with a large proportion of cases being over 50 years. ${ }^{4,10-16,24,27}$ Most patients had a vague prodromal illness of less than a month which is less than 3.7 and 7.1 months reported by others. ${ }^{4,16}$

All patients had evidence of renal involvement; the majority had oliguric renal failure. All had evidence of systemic disease: skin lesions, fever, myalgia, hypertension and lung involvement were common. The clinical features of these patients are similar to other studies of microscopic polyarteritis. ${ }^{4,15}$ The present study is unusual in the high incidence $(61 \%)$ of hypertension. There were no specific haematological or immunological parameters apart from the ANCA, which has been positive in all recent cases. ${ }^{5-7}$

Untreated oliguric acute renal failure due to microscopic polyarteritis has a mortality of $100 \%$. The prognosis can be improved with treatment with steroids, with or without cyclophosphamide. ${ }^{4,10,13-15,19,24,27}$ The first reported survivor of acute renal failure due to PAN was in $1976 .{ }^{17}$

This study suggests that microscopic polyarteritis is 
a wide spectrum of disease. At one end is a group with microscopic haematuria and normal renal function who have a good prognosis and at the other is a severe vasculitis causing a rapid deterioration in renal function requiring dialysis. The severe form of the disease is the commonest in this series and carries a high mortality. Early diagnosis and aggressive treatment with steroids and immunosuppression are essential if the prognosis is to be improved.

It is now our practice to treat new cases of micro-

\section{References}

1. Kussmaul, A. \& Maier, R. Uber eine bisher nicht beschriebene eigenthumliche. Arteriener Krankung (periarteritis nodosa), die mit Morbus Brightii und rapid fortschreitender allgemeiner Muskellahmung einhergeht. Deutsches Arch Klin Med 1866, 1: 484-517.

2. Wainwright, J. \& Davson, J. The renal appearance in the microscopic form of periarteritis nodosa. J Pathol 1950, 62: $189-196$.

3. Davson, J., Ball, J. \& Platt, R. The kidney in periarteritis nodosa. $Q J$ Med 1948, 17: 175-202.

4. Savage, C.O.S., Winearls, C.G., Evans, D.J., Rees, A.J. \& Lockwood, C.M. Microscopic polyarteritis: presentation, pathology and prognosis. $Q J$ Med 1985, 56: 467-483.

5. van der Woude, F.J., Ramussen, N., Lobatto, S. et al. Autoantibodies against neutrophils and monocytes: tool for diagnosis and marker of disease activity in Wegener's granulomatosis. Lancet 1985, i: 425-429.

6. Savage, C.O.S., Winearls, C.G., Jones, S., Marshall, P.D. \& Lockwood, C.M. Prospective study of radioimmunoassay for antibody against neutrophil cytoplasm in diagnosis of systemic vasculitis. Lancet 1987, i: 1389-1393.

7. Venning, M.C., Arfeen, S. \& Bird, A.G. Antibodies to neutrophil cytopolasmic antigen in systemic vasculitis. Lancet 1987, ii: 850 .

8. Fauci, A.S., Haynes, B.F., Katz, P. \& Wolff, S.M Wegener's granulomatosis: prospective clinical and therapeutic experience with 85 patients for 21 years. $A n n$ Intern Med 1983, 98: 76-85.

9. Pinching, A.J., Lockwood, C.M., Pussell, B.A. et al. Wegener's granulomatosis: observations of 18 patients with severe renal disease. $Q J$ Med 1983, 52: 435-460.

10. Serra, A., Cameron, J.S., Turner, D.R. et al. Vasculitis affecting the kidney: presentation, histopathology and long-term outcome. $Q J$ Med 1984, 53: 181-208.

11. Serra, A. \& Cameron, J.S. Clinical and pathological aspects of renal vasculitis. Semin Nephrol 1985, 5: 15-33.

12. Croker, B.P., Lees, T. \& Gunnells, J.C. Clinical and pathological features of polyarteritis nodosa and its renal variant. Hum Pathol 1987, 18: 38-42.

13. Adu, D., Howie, A.J., Scott, D.G., Bacon, P.A., McGonigle, R.J.S. \& Michael, J. Polyarteritis and the kidney. $Q J$ Med 1987, 62: 221-237.

14. Fauci, A.S., Katz, P., Haynes, B.F. et al. Cyclophosphamide therapy of severe systemic necrotizing vasculitis. N Engl J Med 1979, 301: 235-238. scopic polyarteritis initially with prednisolone $60 \mathrm{mg}$ daily and cyclophosphamide $3 \mathrm{mg} / \mathrm{kg} /$ day. Plasmapheresis is currently being evaluated for severe cases.

\section{Acknowledgement}

We are grateful to Dr J.K. Anderson for interpreting the renal biopsies, to Dr F.M. Parsons for access to the records of patients under his care, and to Mrs J. Good for expert secretarial assistance.

15. Coward, R.A., Hamdy, N.A.T., Shortland, J.S. \& Brown, C.B. Renal micropolyarteritis: a treatable condition. Nephrol Dial Transplant 1986, 1: 31-37.

16. Fuiano, G., Cameron, J.S., Raftery, M. Hartley, B.H., Williams, D.G. \& Ogg, C.S. Improved prognosis of renal microscopic polyarteritis in recent years. Nephrol Dial Transplant 1988, 3: 383-391.

17. Tuma, S., Chaimovitz, C., Szylman, P., Gellei, B. \& Better, O.S. Periarteritis in the kidney. Recovery following immunosuppressive therapy. JAMA 1976, 235: $280-281$

18. Leung, A.C.T., McLay, A., Mosley, H. \& BoultonJones, J.M. Polyarteritis group of systemic vasculitis new diagnostic criteria. Scot Med J 1985, 30: 225-231.

19. Lieb, E.S., Restiva, C. \& Paulus, H.C. Immunosuppressive and corticosteroid therapy of polyarteritis nodosa Am J Med 1979, 67: 941-947.

20. Scott, D.G., Bacon, P.A., Elliott, P.J., Tribe, C.R. \& Wallington, T.B. Systemic vasculitis in a District General Hospital 1972-1980: clinical and laboratory features; classification and prognosis of 80 cases. $Q J \mathrm{Med} 1982$, 51: $292-311$

21. Ronco, P., Verroust, P., Mignon, F. et al. Immunopathological studies of polyarteritis nodosa and Wegener's granulomatosis: a report of $\mathbf{4 3}$ patients with 51 renal biopsies. $Q J$ Med 1983, 51: 212-223.

22. Harrison, C.V., Loughridge, L.W. \& Milne, M.D. Acute oliguric renal failure in acute glomerulonephritis and polyarteritis nodosa. $Q J \mathrm{Med} 1964,33: 39-54$.

23. Ladefoged, J., Nielsen, B., Raaschou, F. \& Sorensen, A.W.S. Acute renal failure due to polyarteritis nodosa. Am J Med 1969, 46: 827-831.

24. Cohen, R.D., Conn, D.L. \& Ilstrup, D.M. Clinical features, progress and response to treatment in polyarteritis. Mayo Clin Proc 1980, 55: 146-155.

25. Rose, G.A. \& Spencer, H. Polyarteritis nodosa. Q J Med 1957, 26: 43-79.

26. Zeek, P.M. Periarteritis nodosa: a critical review. Am J Clin Pathol 1952, 22: 777-789.

27. Frohnert, P.P. \& Sheldon, G.S. Long-term follow up study of periarteritis nodosa. Am J Med 1967, 43: 8-14.

28. Fauci, A.S. Vasculitis. J Clin Immunol 1983, 72: 211-223. 